\title{
Blick zurück und Blick nach vorne
}

\author{
Bei der Bezirksversammlung des Freien Verbandes Deutscher Zahnärzte (FVDZ) in Nordwürttem- \\ berg stand auch das 60-jährige Jubiläum der Bezirksgruppe im Fokus. Die Kollegen haben je- \\ doch nicht nur in alten Zeiten geschwelgt, sondern auch die derzeitige Situation der \\ Zahnärzteschaft kritisch beleuchtet.
}

Doch bevor das 60-jährige Bestehen des Freien Verbandes in Baden-Württemberg gefeiert wurde, gab es den offiziellen Teil der Versammlung. Und dort wurde Gerd Hase zum neuen Bezirksvorsitzenden gewählt. Dies war nötig, da die bisherige Bezirksvorsitzende Dr. Gudrun Kaps-Richter im Oktober 2015 zur stellvertretenden FVDZ-Bundesvorsitzenden gewählt wurde und somit nicht mehr für dieses Amt zur Verfügung stand. Neuer stellvertretender Bezirksvorsitzender wurde Dominik Hauk aus Ludwigsburg. Somit rückt zur Freude aller Kollegen ein junger Zahnarzt in den Vorstand auf.

\section{Umfrage über Kosten und Aufwand für Hygiene}

Hase berichtete in seinem Vortrag von der Sitzung der Bezirksgruppenvorsitzenden in Frankfurt. Dort stellte sich der neue FVDZ-Bundesvorstand mit seinem anspruchsvollen Programm für die laufende Amtsperiode vor. Die wichtigsten Punkte erläuterte Kaps-Richter später in ihrem Gastvortrag. Sehr gespannt verfolgten die Zuhörer den Bericht über die vorläufigen Ergebnisse der Umfrage zum Thema Kosten und Aufwand für die Hygiene in der Zahnarztpraxis. Diese Befragung hatte der Bezirksvorstand im Juni durchgeführt. Sie zeigte einige Trends auf: In den Praxen werden erhebliche Investitionen nicht nur, aber zurzeit wohl vor allem im Hygienebereich getätigt. Die Investitionen ziehen massive Folgekosten wie beispielsweise Wartungs- und Validierungskosten nach sich. Im Durchschnitt aller Praxen ist eine zusätzliche Vollzeit-ZFA nötig, um den Hygieneaufwand zu bewältigen. Dabei stellen die Dokumentationspflichten einen erheblichen Posten im Zeitmanagement und damit auch Kostenmanagement dar. Großpraxen liegen mit dreimal so hohen Kosten gegenüber Kleinpraxen deutlich im sechsstelligen Euro-Bereich. Die laufenden Kosten steigen im Jahr 2016 weiter massiv an.

Die ursprünglich mit 50.000 Euro von früheren Untersuchungen kolportierten Kosten werden im Durchschnitt aller Praxen mit Sicherheit deutlich übertroffen. Angesichts dieser TrendUmfrageergebnisse kann eine „Einpreisung“ in die Kassenhonorare im erforderlichen Umfang bisher nicht erfolgt sein. Auch gerade oder wegen den bevorstehenden Begehungen der Praxen in den nächsten Monaten wird dies ein wichtiger Punkt in der Arbeit des FVDZ sein.

\section{Umfangreiches Arbeitsprogramm auf Bundesebene}

In ihrem Gastvortrag hat Kaps-Richter Neues aus dem Bundesvorstand berichtet. Der Bundesvorsitzende Harald Schrader hat ein umfangreiches Arbeitsprogramm aufgestellt. Dauerthema ist die Freiberuflichkeit der Zahnärzte, die es weiter zu wahren gilt. In einem Generationenvertrag sollte das Bündnis Freiberuflichkeit weiter behandelt werden. Der Jugend soll wieder Lust auf Nie- derlassung gemacht werden, wobei die Alten den Jungen mit ihrer Erfahrung die nötige Unterstützung geben sollen. Dabei ist auch an einen Praxispool zur Praxisüberübergabe gedacht.

Im Zusammenhang mit der Freiberuflichkeit spielt auch das Thema MVZ eine große Rolle, da im Moment keine „Waffengleichheit“ mit den klassischen Kooperationsformen besteht. Der FVDZ fordert, dass beide Praxisformen bei der Anstellung von Zahnärzten gleichgestellt werden und lehnt eine Fremdkapitalbeteiligung entschieden ab. Die GOÄ-Novellierung mit robustem Einfachsatz und gefährlichem Paradigmenwechsel im Paragrafenteil wurde zunächst in einem Sonderärztetag abgelehnt, weil FVDZ und BZÄK aufklärten und intervenierten. Es soll aber weiterverhandelt werden. Einzige Änderung ist die Einbeziehung der Facharztverbände. Da die Zahnärzte offenbar als Kollateralschaden abgetan werden, macht sich der FVDZ-Bundesvorstand Gedanken über einen Zukunftsweg Zahnheilkunde, der auch durchaus eine Abkoppelung von der Ärzteschaft in Betracht zieht.

Zur Unterstützung der Kollegenschaft bei den Praxisbegehungen soll ein Leitfaden „Praxisbegehung“ erstellt werden. Dazu ist allerdings zuerst eine Erhebung von belastbarem Datenmaterial nötig. Ein erster Schritt ist eine Rahmenvereinbarung für FVDZ-Mitglieder mit der Firma Valitech.

Mit diesen Informationen hatten die Anwesenden nun einiges zu diskutieren, ohne allerdings die 60-Jahr-Feier inklusive Buffet zu vernachlässigen.

FVDZ-Bezirksgruppe Stutgart

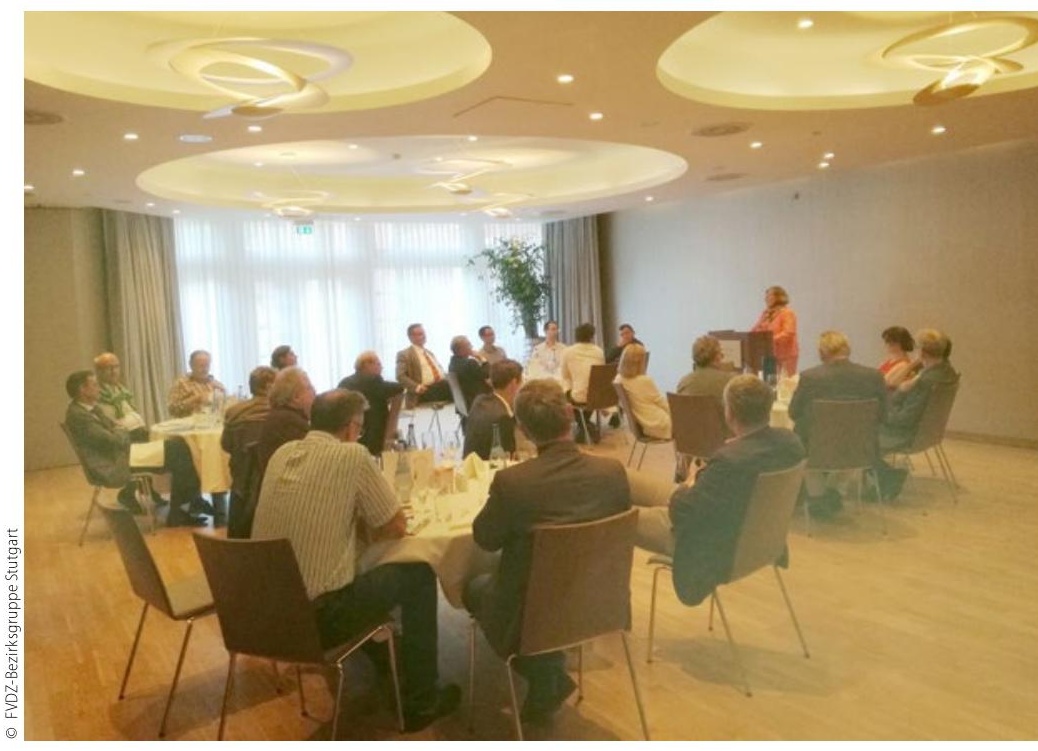

